\title{
Persistent Bleeding Following a Stapled Hemorrhoidopexy
}

\author{
Seong Dae Lee, Sung Taek Jung, Jae-Bum Lee, Mi Jung Kim, Doo-Seok Lee, Eui-Gon Youk, Do-Sun Kim, \\ Doo-Han Lee \\ Departments of Surgery and ${ }^{1}$ Pathology, Daehang Hospital, Seoul, Korea
}

A stapled hemorrhoidopexy (SH) is widely used for treatment of patients with grades III and IV hemorrhoids. The SH is easy to perform, is associated with less pain and allows early return to normal activities. However, complications, whether severe or not, have been reported. Here, we present the case of a female patient with persistent bleeding after a SH. The bleeding was caused by the formation of granulation tissue at the stapler line, diagnosed with sigmoidoscopy, and successfully treated via transanal excision (TAE) under spinal anesthesia. The biopsy showed inflammatory granulation tissue. After the TAE, her symptom was completely gone.

Keywords: Hemorrhoidectomy; Stapled hemorrhoidopexy; Rectal bleeding; Inflammatory polyp

\section{INTRODUCTION}

The stapled hemorrhoidopexy (SH), which can restore a hemorrhoidal cushion, has been widely used for the treatment of patients with hemorrhoids since Dr. Longo [1] reported his experience in 1998. This surgical technique has been shown to be a safe and effective procedure that has several advantages, such as less pain, less discharge, ease of performance, and early return to normal activities $[2,3]$. However, like other surgical techniques, it has been reported to have a variety of complications from minor to life threatening. The serious complications that have been reported in the literature may have been caused by a lack of experience by those performing this procedure $[4,5]$. However, for any surgeon who treats patients with hemorrhoids, minor complications can occur with an incidence rate around 25\% [3]. In rare cases, an inflammatory polyp that causes rectal bleeding and is discovered incidentally following a SH has been reported. Here, we present a case with an uncommon complication following a $\mathrm{SH}$.

Received: August 28, 2015 - Accepted: March 16, 2016

Correspondence to: Seong Dae Lee, M.D.

Department of Surgery, Daehang Hospital, 2151 Nambusunhwan-ro,

Seocho-gu, Seoul 06699, Korea

Tel: +82-2-6388-8114, Fax: +82-2-6388-8673

E-mail:vocalcord@hanmail.net

(C) 2016 The Korean Society of Coloproctology

This is an open-access article distributed under the terms of the Creative Commons Attribution NonCommercial License (http://creativecommons.org/licenses/by-nc/4.0) which permits unrestricted noncommercial use, distribution, and reproduction in any medium, provided the original work is properly cited.

\section{CASE REPORT}

A 59-year-old female presented with a third to fourth degree hemorrhoid, complaining of anal prolapse during defecation. Upon digital rectal examination, the anal sphincter tone was weak, and a third to fourth grade hemorrhoid was diagnosed. The patient preoperatively underwent a clinical examination and routine laboratory test. A colonoscopy one year earlier had been normal. A SH was performed in the prone jackknife position under spinal anesthesia by using a HEM 3335 unit (Covidien, Mansfield, MA, USA). She was normally discharged on the second postoperative day as scheduled.

An office visit was scheduled for one week and three weeks after surgery. She had frequent bowel movements, as often as 5 to 6 times a day. An intermittent tearing sensation during defecation disappeared after 3 months. She complained of a recurrent small amount of bleeding at postoperative month 8. Anoscopy failed to demonstrate a bleeding focus because of a large amount of stool, so we decided that a further evaluation, such as a sigmoidoscopy, was needed. Flexible sigmoidoscopy showed two 8-mm-sized friable, sessile masses at the previous operation site (Fig. 1A). A transanal excision (TAE) under spinal anesthesia was performed to exclude a malignancy and to treat the bleeding tendency (Fig. 1B). Pathologic examination showed the formation of nonneoplastic crypts and abundant inflamed stroma, suggesting an inflammatory polyp (Fig. 2). After the TAE, her symptoms were completely resolved. A follow-up anoscopy one month later demonstrated no recurrence of polyps. 

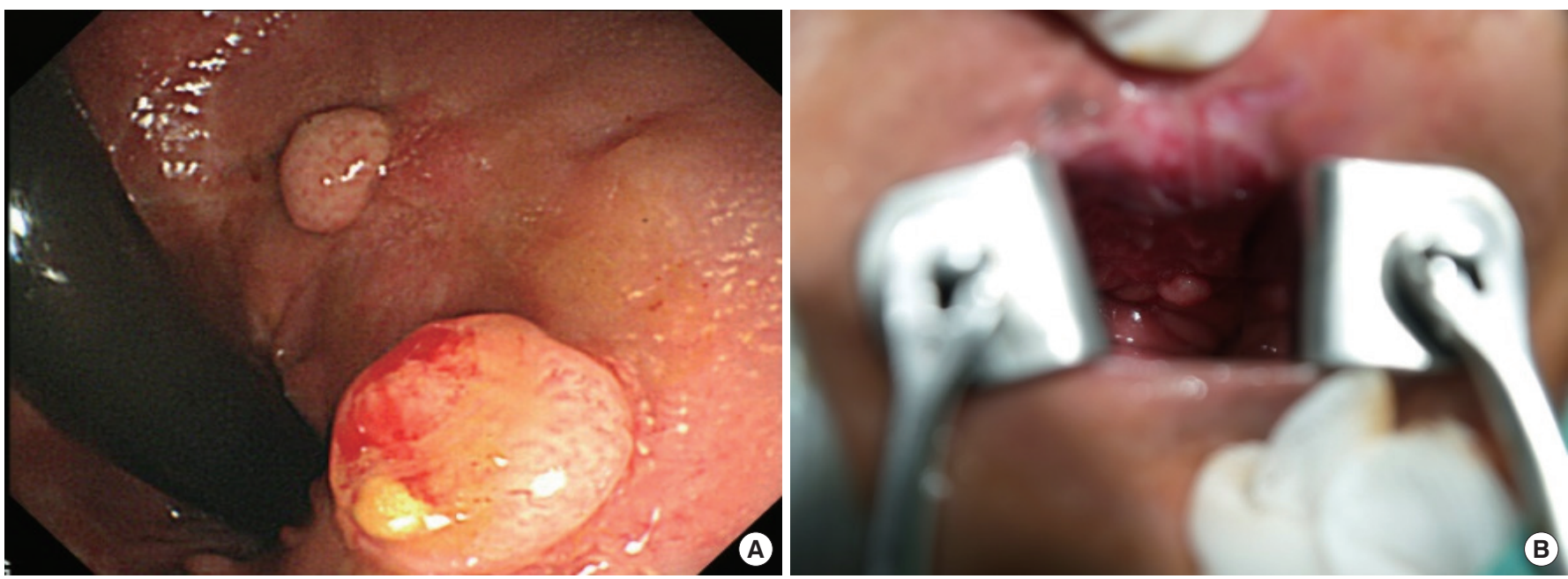

Fig. 1. (A) Endoscopic findings of polyps after a staple hemorrhoidopexy: 8-mm-sized sessile polyps at the location of the scar from previous surgery, and (B) operative finding of the polyps: 2 friable polyps, 1 each in the 6 and 8 oclock directions.

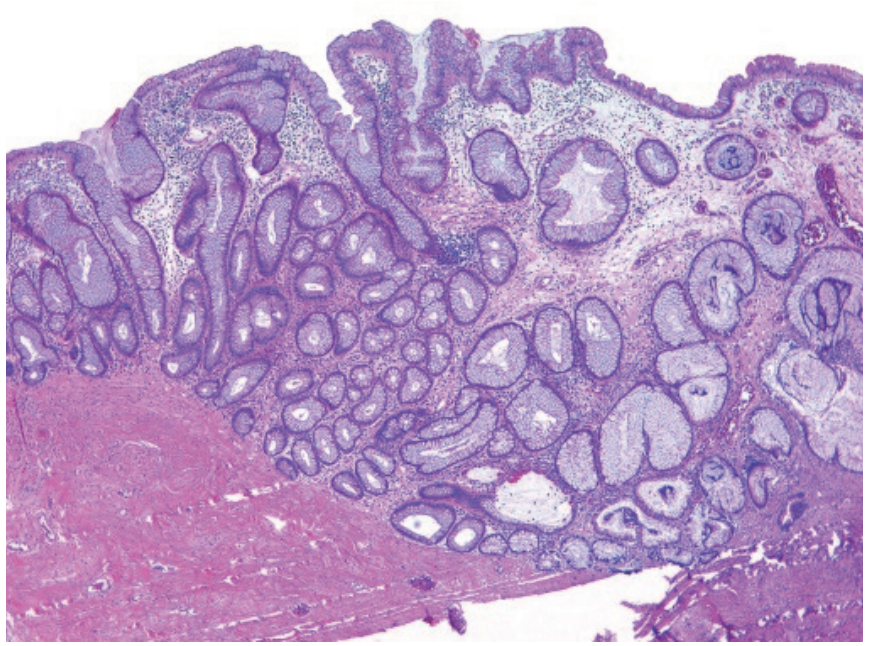

Fig. 2. Histological image of the resected polyps consisting of nonneoplastic crypts and abundant inflamed stroma, suggesting an inflammatory polyp $(\mathrm{H} \& \mathrm{E}, \times 40)$.

\section{DISCUSSION}

A hemorrhoid is caused by a pathologic change in the anal cushion involving the destruction of Parks' ligament $[6,7]$. A SH is an ideal surgical technique for restoring the anal cushion through a resection and repositioning of a redundant hemorrhoid pile. However, like other surgical techniques, it has a variety of reported complications, some of which, such as pelvic sepsis, a rectal hematoma, perforation, and pneumomediastinum, may be life threatening. However, theses serious complications are rare. On the contrary, minor complications, such as bleeding, pain, stenosis, and prolapse, are common after a SH. Therefore, many sur- geons experience such minor complications more frequently than they experience serious complications.

Of these complications, postoperative bleeding occurs early, mostly within 2 weeks following surgery, and bleeding following a $\mathrm{SH}$ is estimated to occur in $4.2 \%-7.5 \%$ of the cases [8]. Surgical reintervention for postoperative bleeding is necessary in approximately $0.4 \%-1 \%$ of the cases. Although life-threatening bleeding has been reported, most cases involve minor, self-limiting luminal bleeding that arises from the arteriolar vessels of the submucosa along the staple line. However, if bleeding symptoms persist after this period, other causes of bleeding, such as the occurrence of an anal fissure, fistula, remnant staple, or abnormal colon condition, should be considered.

Overall, inflammatory polyps are believed to arise secondarily to ulceration and repair [9]. However, in cases after a SH, the exact mechanism for an inflammatory polyp to develop is unclear. Several factors may influence the formation of an inflammatory polyp after a SH. For example, mechanical factors such as repeated injury and repair, as well as remnant staples, may cause an inflammation and result in the formation of polyps. If an inflammatory polyp after a $\mathrm{SH}$ is caused by chronic irritation around a remnant staple, bleeding may be treated by removing the remnant staple. Quah et al. [10] reported 2 cases of bleeding at 12 months after a stapled hemorrhoidectomy. In those cases, the bleeding disappeared after the staples had been removed. Fondran et al. [11] reported 9 cases of bleeding between 6 and 16 weeks after the procedure. All polyps were located at the staple line. The problem could not be resolved using topical silver nitrate. Excision of the polyps did result in cessation of the bleeding, and the polyps did not recur after their excision. However, in our case and the cases of Fondran et al. [11], because no remnant staple was found at the polyps, secondary ulceration and foreign body reaction of suture materials at the staple line was probably the cause of the inflam- 
matory polyp. Therefore, chronic intramural stimulation during defecation may be a second possible cause for the formation of the rectal inflammatory granulation polyp, as in cases of silk sutures and vegetable seeds in colonic polyps $[12,13]$. However, Fondran et al. [11] reported that in 2 of 9 patients, a polyp formed without suture reinforcement. Therefore, chronic intramural stimulation by a foreign body may not totally explain the formation of an inflammatory polyp. In our case, no staples or suture materials were found at the polyps. Therefore, we hypothesize that the formation of the inflammatory polyp in our case was caused by frequent bowel movement, which could induce a minor intramural injury.

Although data on the clinical course of and nonsurgical therapy for an inflammatory polyp are lacking, reports indicate that it rarely regresses with nonsurgical therapy alone [10] and may require a surgical or endoscopic resection to control the bleeding. Weinstock and Shatz [14] reported a benign inflammatory polyp at the anastomosis site after a colon-cancer resection. They excised the inflammatory polyp with forceps or a snare. We totally agree with the result of that previous study and recommend that any remnant staples or suture material be removed. Also, if symptomatic polyps exist along the staple line, with or without remnant staples and/or suture material, they should be removed.

This complication of an inflammatory polyp should be suspected if signs and symptoms of bleeding, either a small amount of bleeding or persistent bleeding, develop postoperatively. We recommend that an anoscopy or sigmoidoscopy should be considered to detect such a condition.

In conclusion, we report a case of recurrent bleeding following a $\mathrm{SH}$, which necessitated a surgical resection to alleviate the patient's symptoms. In case of bleeding for a long duration after a $\mathrm{SH}$, bleeding caused by an inflammatory polyp, although very rare, should be considered. The correct application of the procedure and an understanding of the proper indications, the anatomy, and possible complications are essential if such complications are to be prevented.

\section{CONFLICT OF INTEREST}

No potential conflict of interest relevant to this article was reported.

\section{REFERENCES}

1. Longo A. Treatment of haemorrhoid disease by reduction of mucosa and haemorrhoidal prolapse with a circular-suturing device: a new procedure. In: Proceedings of the Sixth World Congress of Endoscopic Surgery; 1998 Jun 3-6; Rome, Italy. Bologna: Monduzzi Editore, International Proceedings Division, 1998. p. 777-84.

2. Giordano P, Gravante G, Sorge R, Ovens L, Nastro P. Long-term outcomes of stapled hemorrhoidopexy vs conventional hemorrhoidectomy: a meta-analysis of randomized controlled trials. Arch Surg 2009;144:266-72.

3. Mehigan BJ, Monson JR, Hartley JE. Stapling procedure for haemorrhoids versus Milligan-Morgan haemorrhoidectomy: randomised controlled trial. Lancet 2000;355:782-5.

4. Herold A, Kirsch JJ. Pain after stapled haemorrhoidectomy. Lancet 2000;356:2187.

5. Pavlidis T, Papaziogas B, Souparis A, Patsas A, Koutelidakis I, Papaziogas T. Modern stapled Longo procedure vs. conventional Milligan-Morgan hemorrhoidectomy: a randomized controlled trial. Int J Colorectal Dis 2002;17:50-3.

6. Brisinda G. How to treat haemorrhoids. Prevention is best; haemorrhoidectomy needs skilled operators. BMJ 2000;321:582-3.

7. Thomson WH. The nature of haemorrhoids. Br J Surg 1975;62: 542-52.

8. Kim JS, Vashist YK, Thieltges S, Zehler O, Gawad KA, Yekebas EF, et al. Stapled hemorrhoidopexy versus Milligan-Morgan hemorrhoidectomy in circumferential third-degree hemorrhoids: long-term results of a randomized controlled trial. J Gastrointest Surg 2013;17:1292-8.

9. Watne AL. Colon polyps. J Surg Oncol 1997;66:207-14.

10. Quah HM, Hadi HI, Hay DJ, Maw A. Residual staples as a possible cause of recurrent rectal bleeding after stapled haemorrhoidectomy. Colorectal Dis 2003;5:196.

11. Fondran JC, Porter JA, Slezak FA. Inflammatory polyps: a cause of late bleeding in stapled hemorrhoidectomy. Dis Colon Rectum 2006;49:1910-3.

12. Araki Y, Akahoshi K, Harada N, Chijiiwa Y, Sasaki I, Nawata H. Two cases of colonic adenomatous polyps accompanied by a migrated surgical suture. Endoscopy 2001;33:85-7.

13. Velanovich V. Vegetable seed simulating colon polyp. Surg Endosc 1995;9:456.

14. Weinstock LB, Shatz BA. Endoscopic abnormalities of the anastomosis following resection of colonic neoplasm. Gastrointest Endosc 1994;40:558-61. 